\title{
TITLE:
}

\section{Ultrasonic Velocity and Adiabatic Compressibility of Mixture of Sulfuric Acid and Water}

$\operatorname{AUTHOR}(S)$ :

Ishida, Yoshio

CITATION:

Ishida, Yoshio. Ultrasonic Velocity and Adiabatic Compressibility of Mixture of Sulfuric Acid and Water. Memoirs of the College of Science, University of Kyoto. Series A 1958, 29(1): $91-105$

\section{ISSUE DATE:}

1958-09

URL:

http://hdl.handle.net/2433/257422

RIGHT: 


\title{
Memoirs of the College of Science, University of Kyoto, Series A, Vol. XXIX, No. 1, Article 8, 1958. \\ ULTRASONIC VELOCITY AND ADIABATIC COMPRESSIBILITY OF MIXTURE OF SULFURIC ACID AND WATER*
}

\author{
BY \\ Yoshio ISHIDA*** \\ (Received June 18, 1958)
}

\section{Introduction}

Ultrasonic velocities in various liquid mixtures have been measured by several experimenters. We have carried out a measurement on mixtures of sulfuric acid and water at $5 \mathrm{Mc} / \mathrm{sec}$. After finishing it, the author was informed of Prosorow's result (1) and was very glad to have an opportunity to make comparison. In the present research, the ultrasonic velocity in aqueous solution of the sulfuric acid was measured by use of the rotary grating method $(2,3,4)$ which is a variation of the Debye-Sears light diffraction method (5).

The rotary grating method is, in principle, a method to measure, by rotating an optical grating, the distance between diffraction lines which are generated by ultrasonic waves in liquid. It has a higher accuracy than the method to measure directly the diffraction angle.

Moreover, since the present apparatus has not any metallic parts in its sample container, it has become easy to measure ultrasonic velocity in strong acids such as sulfuric acid.

\section{Experimental arrangement}

The experimental arrangement is shown in Fig. 1. Since a new apparatus has been improved a little as compared with the old one used in the previous research (4), a brief description of it will be given here.

A light beam is obtained from a sodium lamp by a collimator having a horizontal slit. It is diffracted doubly by ultrasonic waves in a sample and a rotary grating. The diffraction images generated can be observed by a telescope. In order to observe the diffraction image at its weakest intensity, the plate voltage of power amplifier is made adjustable by means of a slidac. The sample container is provided with a

* Communicated by Professor I. Takahashi. A short account of the present paper was already given in J. Phys. Soc. Japan, 13 (1958), 636.

** Department of Physics, Faculty of Education, Shimane University, Matsue. 
water-jacket around it in order to control the temperature of sample liquid. The water from a constant temperature bath flows through the water-jacket and the wave generating tank and enters a reservoir, from which the water is returned again to the constant temperature bath by a circulation pump. The temperature of water in the bath is kept constant by a mercury regulator and a heater. The optical grating is fixed in a cylinder which can be rotated with an angle measuring disk attached, to serve as a rotary grating. The proper constant of the grating is chosen to be $2.000 \times 10^{-3} \mathrm{~cm}$. The circumference of the disk is divided into 360 equal parts, and the angle of rotation can be measured correct to two minutes by an attached vernier.

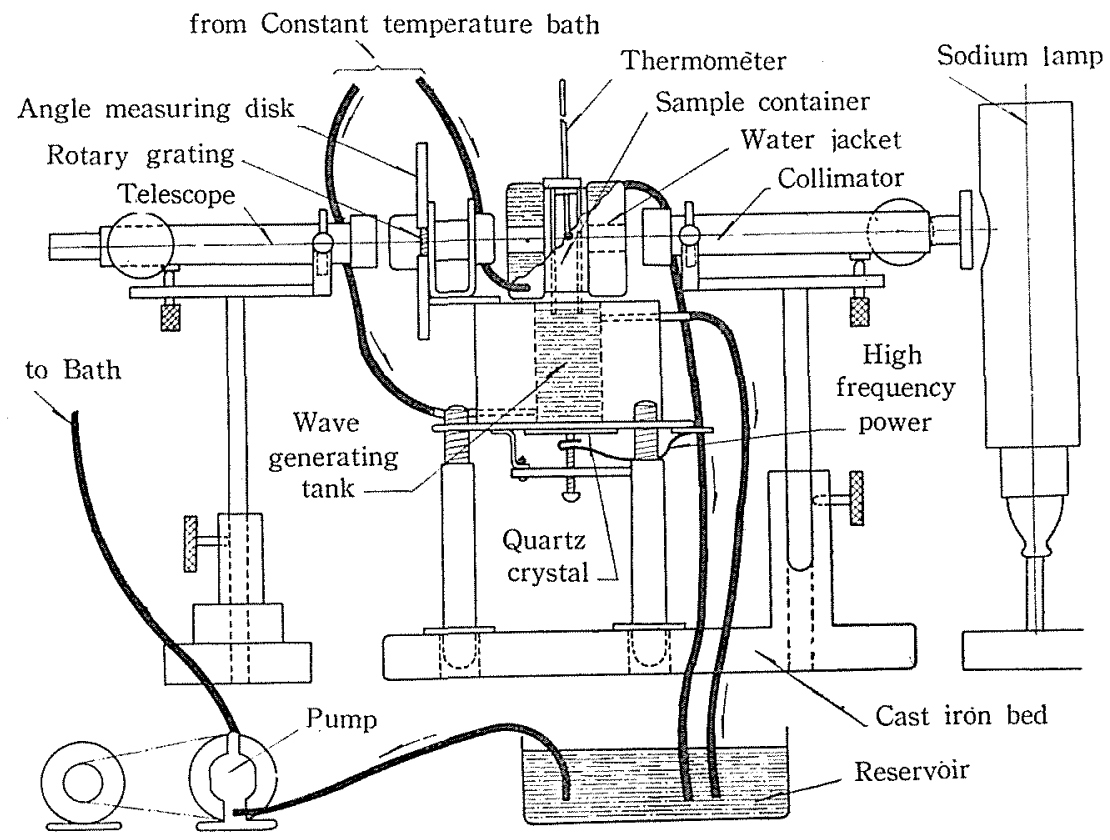

Fig. 1. Experimental arrangement.

\section{Method of measuring ultrasonic velocity}

Various images seen in the visual field of telescope are shown in Fig. 2. The method of measurement is explained in connection with them. Figure (a) shows an image seen when there are no ultrasonic waves and the lines of rotary grating are perpendicular to the slit. Only the image of slit appears which may be called the main line. Figure (b) shows an appearance when the grating is rotated from there. The first order diffraction lines by the grating appear on both sides of the main line and move with the rotation of the grating. They may be called the rotary main line. 


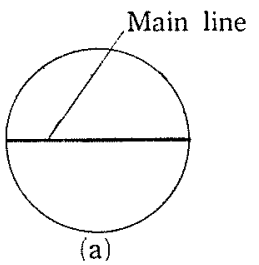

No waves, lines of No waves, grating grating perpendicular rotated from (a) to slit

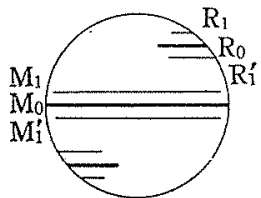

(e)

Waves present, grating rotated

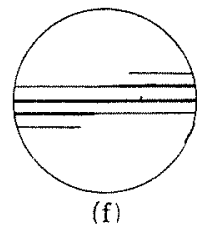

First order coincidence

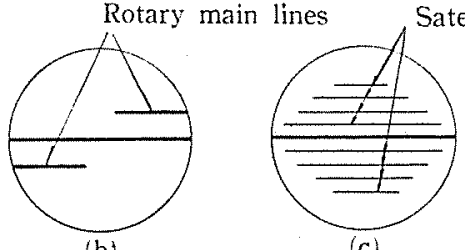

Waves applied to (a)
First order Satellite lines satellite lines

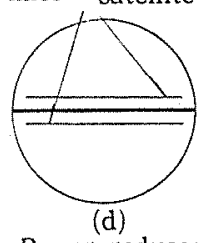

Power reduced from $(c)$

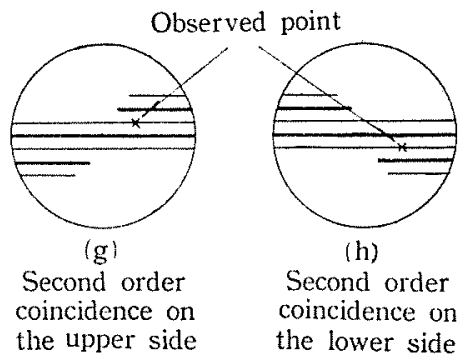

Fig. 2. Diffraction images seen by telescope.

The distance between the main line and the rotary main line becomes maximum when the line of grating is made parallel to the slit. By measuring the distance and the angle of rotation of the grating, it was experimentally ascertained (1) that the formula

$$
d=\frac{d_{0}}{\sin \theta}
$$

should hold between the effective constant of the grating $d$ and its proper constant $d_{0}$.

When the ultrasonic waves are applied to the state of figure (a), several diffraction lines by the waves appear as shown in figure (c). They may be called the satellite lines. Since it is preferable to make measurement with possibly weakest power, the power is reduced to such an extent that only the first order satellite lines remain in the visual field as shown in figure (d). When the grating is rotated from the state of figure (d), the rotary main lines move, being accompanied by their respective first order satellite lines as shown in figure (e). On the way of rotation, the diffraction lines make some coincidence with each other. Figure (f) shows the case in which the rotary main lines coincide with the satellites of the main line. The case may be called the first order coincidence. Figure $(\mathrm{g})$ shows the case in which the lower satellite of the rotary main line coincides with the upper satellite of the main line. The case may be called the second order coincidence on the upper side. Similarly, figure $(\mathrm{h})$ shows the case of the second order coincidence on the lower side. Since the main lines are thick and the satellites fine, it gives more 
accurate result to observe the coincidence between satellites themselves than to observe that between the rotary main line and the satellite. Therefore, the second order coincidence was used in the measurement.

Now, if $\theta_{\mathrm{U}}$ is the reading of the coincidence angle in figure $(\mathrm{g})$ and $\theta_{\mathrm{L}}$ is that in figure $(\mathrm{h}), \theta=\frac{1}{2}\left(\theta_{\mathrm{U}}-\theta_{\mathrm{L}}\right)$ should serve as $\theta$ in the formula (1). Considering that diffraction lines coincide with each other when the wavelength of the ultrasonic wave becomes an integral multiple of the effective constant of the grating $d$, we obtain

$$
\Lambda=n d,
$$

where $A$ is the wavelength of the ultrasonic wave and $n$ a positive integer.

If $f$ is the frequency of the ultrasonic wave, we get, from (1) and (2), the formula for the velocity $V$ as:

$$
V=f \Lambda=\frac{n d_{0} f}{\sin \theta}
$$

In the present case, putting $d_{0}=2 \times 10^{-3} \mathrm{~cm}, f=5 \times 10^{6} \mathrm{c} / \mathrm{s}$ and $n=2$ we obtain

$$
V=\frac{200}{\sin \theta}[\mathrm{m} / \mathrm{sec}]
$$

The error of the measurement is considered as follows. The readings of the coincidence angle are generally different according as the grating is rotated clockwise or counter-clockwise. In figure (e), if

$\theta_{\mathrm{U} 1}$ is the reading when $R_{1}^{\prime}$ line approaches $M_{1}$ from above and coincides with it, $\theta_{\mathrm{U} 2}$ the reading when $R_{1}^{\prime}$ line approaches $M_{1}$ from below and coincides with it, $\theta_{\mathrm{L} 1}$ the reading when $R_{1}$ line approaches $M_{1}^{\prime}$ from above and coincides with it, $\theta_{\mathrm{L} 2}$ the reading when $R_{1}$ line approaches $M_{1}^{\prime}$ from below and coincides with it, the required angle readings $\theta_{\mathrm{U}}, \theta_{\mathrm{L}}$ and $\theta$ are obtained by

and

$$
\begin{aligned}
& \theta_{\mathrm{U}}=\theta_{\mathrm{U} 2}+\frac{1}{2}\left(\theta_{\mathrm{U} 1}-\theta_{\mathrm{U} 2}\right)=\theta_{\mathrm{U} 2}+\Delta \theta_{\mathrm{U}}, \\
& \theta_{\mathrm{L}}=\theta_{\mathrm{L} 2}+\frac{1}{2}\left(\theta_{\mathrm{L} 1}-\theta_{\mathrm{L} 2}\right)=\theta_{\mathrm{L} 2}+\Delta \theta_{\mathrm{L}}, \\
& \theta=\frac{1}{2}\left(\theta_{\mathrm{U}}-\theta_{\mathrm{L}}\right)=\frac{1}{2}\left(\theta_{\mathrm{U} 2}-\theta_{\mathrm{L} 2}\right)+\frac{1}{2}\left(\Delta \theta_{\mathrm{U}}-\Delta \theta_{\mathrm{L}}\right) .
\end{aligned}
$$

Therefore, $\Delta \theta=\frac{1}{2}\left(\left|\Delta \theta_{\mathrm{U}}-\Delta \theta_{\mathrm{L}}\right|\right)$ is considered as a measure for determining the accuracy of the apparatus.

If we repeat the measurements very many times, $\left\langle\Delta \theta_{\mathrm{U}}\right\rangle$ will become equal to $\left\langle\Delta \theta_{\mathrm{L}}\right\rangle$, but $\left\langle\left|\Delta \theta_{\mathrm{U}}-\Delta \theta_{\mathrm{L}}\right|\right\rangle$ will take a definite value. An example of the measurements is given below. 
Example: $\quad$ Sample:- Distilled water $\left(30^{\circ} \mathrm{C}\right)$

$$
\begin{aligned}
& \text { Upper coincidence }\left\{\begin{array}{lll}
\theta_{\mathrm{U} 1} & 222^{\circ} & 46^{\prime} \\
\theta_{\mathrm{U} 2} & 222^{\circ} & 34^{\prime}
\end{array}\right. \\
& \text { Difference } 12^{\prime} \cdots \Delta \theta_{U}=6^{\prime} \\
& \text { Lower coincidence }\left\{\begin{array}{lll}
\theta_{L_{1}} & 207^{\circ} & 30^{\prime} \\
\theta_{\mathrm{L} 2} & 207^{\circ} & 20^{\prime}
\end{array}\right. \\
& \text { Difference } 10^{\prime} \cdots \Delta \theta_{\mathrm{L}}=5^{\prime} \\
& \text { and } \theta=7^{\circ} \quad 38^{\prime} \text {. }
\end{aligned}
$$

Calculating with the values of $\Delta \theta_{\mathrm{U}}, \Delta \theta_{\mathrm{L}}$ and $\left|\Delta \theta_{\mathrm{U}}-\Delta \theta_{\mathrm{L}}\right|$ which were obtained in the series of 967 measurements during the period of the present research, we get

$$
\left\langle\Delta \theta_{\mathrm{U}}\right\rangle=8.31^{\prime},\left\langle\Delta \theta_{\mathrm{L}}\right\rangle=7.11^{\prime} \text { and }\left\langle\left|\Delta \theta_{\mathrm{U}}-\Delta \theta_{\mathrm{L}}\right|\right\rangle=4.54^{\prime} \text {. }
$$

Thus, putting $\Delta \theta=\frac{1}{2}\left\langle\left|\Delta \theta_{\mathrm{U}}-\Delta \theta_{\mathrm{L}}\right|>=2.27^{\prime}\right.$ in the error formula :

$$
\Delta V=-n d_{0} f \frac{\cos \theta}{\sin ^{2} \theta} \frac{\pi}{180} \Delta \theta
$$

which can be derived from the formula (3), we get $\Delta V=2.97[\mathrm{~m} / \mathrm{sec}]$, for the distilled water under consideration.

This error corresponds to the accuracy of about $2 / 1,000$ and may be called the proper error of the apparatus. And $\left\langle\Delta \theta_{\mathrm{U}}\right\rangle,\left\langle\Delta \theta_{\mathrm{L}}\right\rangle$ may be called the angular line width, and $\frac{1}{2}<\left|\Delta \theta_{\mathrm{U}}-\Delta \theta_{\mathrm{L}}\right|>$ the proper error angle. These values will serve as criterions for the purpose of reducing the error when the apparatus will be improved in a future. It is considered that the angular line width depends principally upon the width of slit, the light intensity, the ultrasonic intensity, etc. And, on the other hand, the proper error angle depends upon the deviation from symmetry of the light and ultrasonic system.

Since the proper error $2 / 1,000$ is a little too large for our purpose of the experiment, the mean value from fifteen measurements was taken as the significant value of the velocity in the sample with a certain concentration and temperature. Therefore, the probable error of the final value was calculated to be $\pm 0.54 \mathrm{~m} / \mathrm{sec}$.

\section{Experimental results}

\section{Comparison with Prosorow's data}

The ultrasonic velocity in the aqueous solution of the sulfuric acid was measured versus concentration. After finishing it, the author was informed of the publication of Prosorow's data. So the author's data are shown in Fig. 3 in comparison with his ones. The concentration is expressed in weight percentage, following Prosorow. 
From the curves in Fig. 3, it will readily be seen that in the lower concentration region, both curves coincide fairly well, while in the medium concentration region, the two curves are quite similar, the author's curve lying by about $10[\mathrm{~m} / \mathrm{sec}]$ below Prosorow's, and the positions of maxima coincide, situated at $45 \%$. In the higher concentration region, the difference between the two curves becomes larger and ultimately attains the values of $29[\mathrm{~m} / \mathrm{sec}]$ at $91 \%$. In this region, however, the

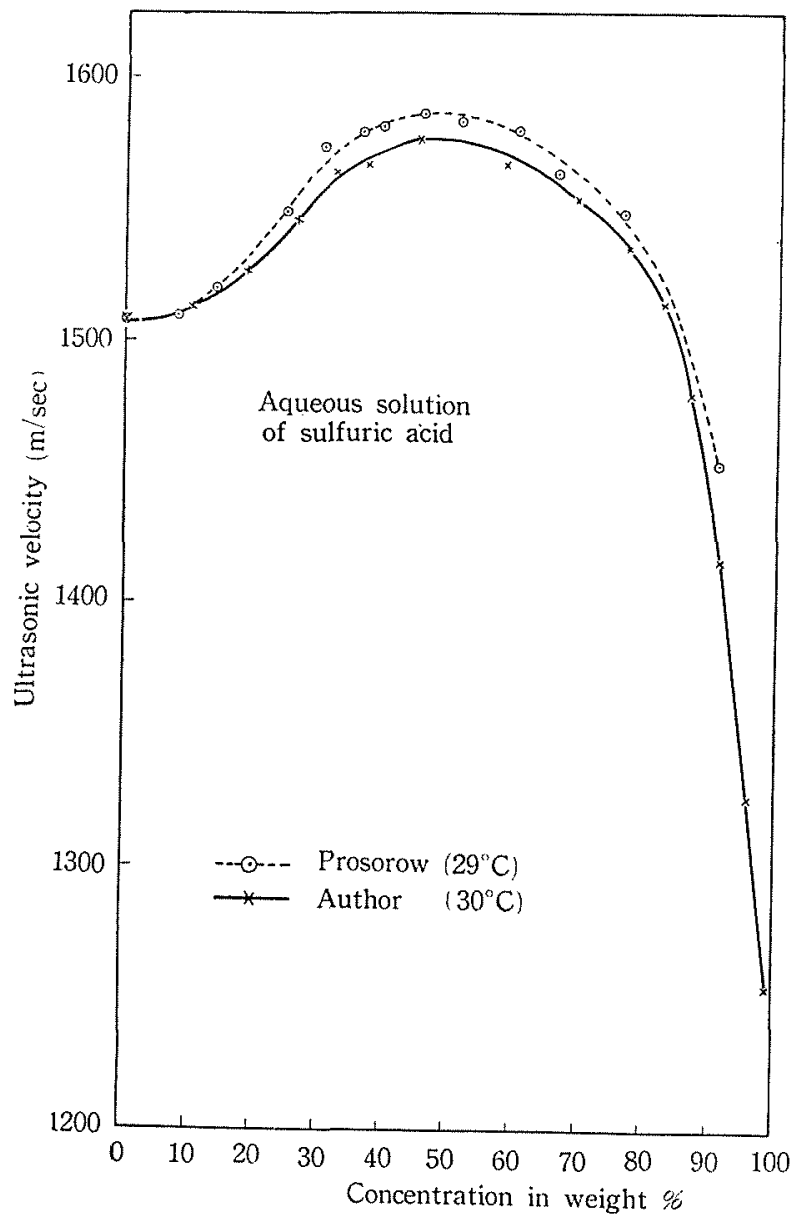

Fig. 3. Comparison with Prosorow's data.

difference of this order of magnitude might be inevitable, since the velocity decreases very rapidly with the increasing concentration-the velocity decrease of $20[\mathrm{~m} / \mathrm{sec}]$ corresponding to 196 increase in concentration. The sulfuric acid of the special class was used as a sample, and especially in order to prepare a sample of high concentration of $99.3 \%$, the sulfuric acid was mixed with fuming sulfuric acid. 


\section{Velocity and compressibility versus concentration characteristics at two temperatures}

The author measured the ultrasonic velocity versus concentration at two different temperatures. The results, together with the adiabatic compressibility calculated from them, are shown in Fig. 4. The concentration is expressed in this case in mole fraction of sulfuric acid which is considered to be more convenient for the consideration of the molecular structure of the liquid than in weight percentage. To get the value of concentration, the normality of the solution is measured by acidimetry. It is possible to obtain from tables the values of density of the solution at $15^{\circ} \mathrm{C}$ and $30^{\circ} \mathrm{C}$ corresponding to the values of normality at which measurements were done. We can calculate the mole fraction of the sulfuric acid from the normality and the density. The adiabatic compressibility can be computed by $1 / \rho V^{2}$, where $V$ is the measured velocity and $\rho$ the density.

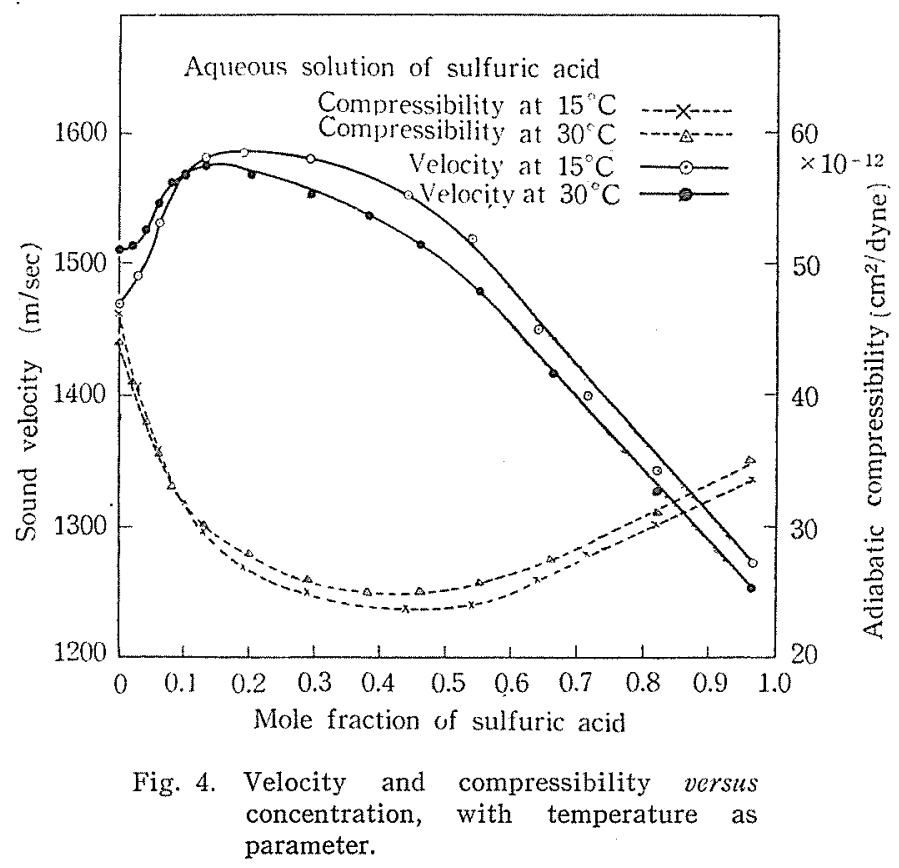

From Fig. 4 it will readily be seen that the velocity-curves have maxima, while the compressibility-curves minima. The position of maximum of the velocity-curve shifts to the higher concentration as the temperature decreases. The position of minimum of the compressibility curve does not shift appreciably, but the value of minimum decreases with the temperature. The results will be discussed in the next section. 


\section{Velocity-temperature characteristic at several concentrations}

The ultrasonic velocity in the solution was measured in the temperature range covering from $6^{\circ} \mathrm{C}$ to $46^{\circ} \mathrm{C}$, with concentration as parameter. The result is shown in Fig. 5. In the higher concentration, the curves become straight with negative gradient. From the straight lines V, VI and VII, the velocity-temperature coefficient is calculated to be $-1.61 \mathrm{~m} / \mathrm{sec} / \mathrm{deg}$ at $0.437 \mathrm{mf},-1.53 \mathrm{~m} / \mathrm{sec} / \mathrm{deg}$ at $0.715 \mathrm{mf}$,

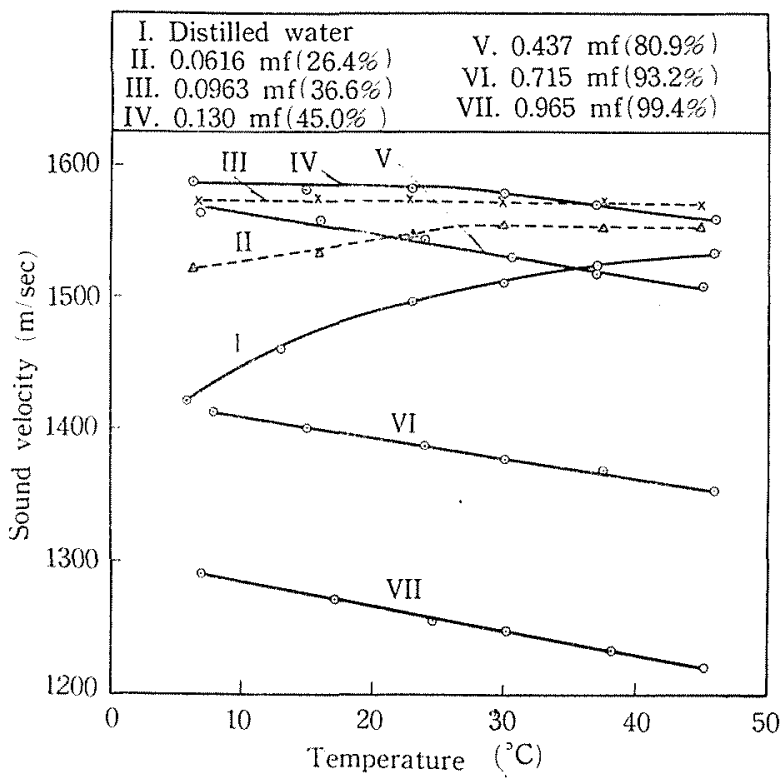

Fig. 5. Velocity versus temperature, with concentration as parameter.

and $-1.80 \mathrm{~m} / \mathrm{sec} / \mathrm{deg}$ at $0.965 \mathrm{mf}$. It should be noted that as seen from the straight line III, the velocity is independent of the temperature at the concentration of $0.0963 \mathrm{mf}$. The curve $I$ is the characteristic of the distilled water which is measured for comparison and found to be parabolic as known already. The discussion about the results will be given in the next section.

\section{Discussion}

\section{Regarding the compressibility-concentration characteristic}

The phenomenon that the velocity-curve has maximum as shown in Fig. 4 is common to all of the mixtures of methyl alcohol-water (6), ethyl alcohol-water (7), acetone-water (7), pyridine-water (6), formic acid--water (8), acetic acid-water $(6,9)$, nitric acid-water (1), carbon tetrachloride-liquid paraffin (10) and others. We ascertained as shown in Table $I$ that the volume contraction always takes place when these mixtures are prepared, including the mixture of sulfuric acid and water. 
Table I. Index of volume contraction and heat of dilution in liquid mixture.

\begin{tabular}{l|c|c|c|c}
\hline \multirow{2}{*}{ Mixture } & \multicolumn{2}{|c|}{ Index of volume contraction } & \multicolumn{2}{c}{ Heat of dilution } \\
\cline { 2 - 5 } & $t^{\circ} \mathrm{C}$ & $-\Delta V / V \times 100^{*}$ & $t^{\circ} \mathrm{C}$ & $Q^{* *}$ \\
\hline Methyl alcohol-Water & 10.0 & 4.00 & 10 & 8.38 \\
Ethyl alcohol-Water & 12.0 & 4.52 & 13 & 10.63 \\
Aceton-Water & 14.5 & 3.92 & 10 & 10.50 \\
Pyridine-Water & 11.0 & 2.40 & 22 & 8.87 \\
Formic acid-Water & 12.0 & 2.26 & 7 & 0.33 \\
Acetic acid-Water & 9.0 & 2.68 & 23 & 1.00 \\
Nitric acid-Water & 12.0 & 3.26 & - & - \\
Sulfuric acid-Water & 30.0 & 7.60 & - & - \\
Carbon tetrachloride- & 12.0 & 3.20 & - & - \\
Liquid paraffin & & & & - \\
\hline
\end{tabular}

* $\Delta V$ is the volume contraction when component liquids are mixed in equal volumes. $V$ is the sum of volumes of component liquids.

** When water is considered as solvent, $Q$ is expressed in kilo-joules per mole solute at infinite dilution (International Critical Tables, Vol. 5, p. 148).

Therefore, there exists a relation between the volume contraction and the existence of maximum in the velocity-curve and of minimum in the compressibility-curve. Ernst (10) has pointed out that the solutions which show this characteristic do not obey Raoult's law. We want to examine the idea more closely. The solutions which do obey Raoult's law are the so-called ideal solutions and they have no heat of dilution and there is no volume change when the components are mixed in liquid state. The pairs of liquids listed in Table I are all considered to make non-ideal solutions, as they have heat of dilution* and there occur volume changes when mixed.

It is well known that there are two types of non-ideal solution in connection with the deviation from Raoult's law. One is a positive deviation type as shown in Fig. 6 (a)**. This is the case when the escaping tendency of A molecules is increased by the presence of $B$ molecules, since the attraction between $B$ molecules is much greater than that between $A$ molecules. The other is a negative deviation type as shown in Fig. 6 (b)**. The case occurs when there exists the strong attraction between $\mathrm{A}$ and $\mathrm{B}$ molecules. In both figures, dotted-line curves show ideal behaviors.

When we observe the compressibility characteristic of the non-ideal solutions cited above, keeping the theory in mind, we become aware of a similarity between both characteristics of compressibility and total vapor pressure. It is certain that the

* The approvable data of heat of dilution concerning the last three mixtures in Table I could not be found. But its existence is evident from our simple experiences.

*** These figures are quoted from: S. Glasstone: Elements of Physical Chemistry (1954), p. 356. 
existence of the compressibility minimum which is, more generally, the negative deviation from the ideal behavior, comes from the attraction between the component molecules. Therefore, they are expected also to show the negative deviation from Raoult's law.

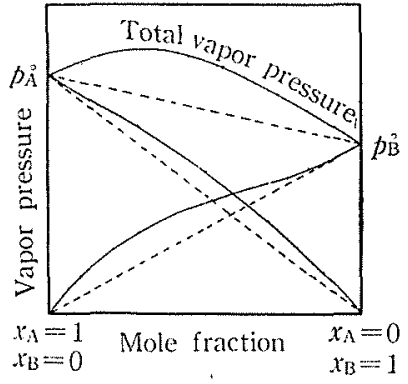

(a)

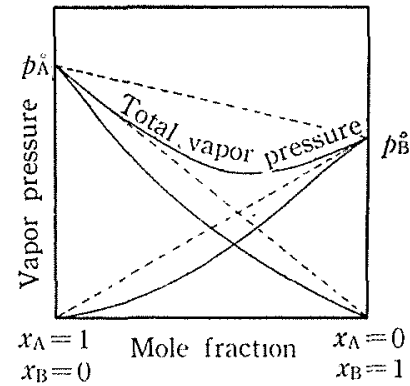

b

Fig. 6. Total vapor pressure versus concentration of non-ideal solutions.

In the case of the aqueous solution of sulfuric acid, the hydronium ion $\left(\mathrm{H}_{3} \mathrm{O}\right)^{+}$ and sulfuric acid ion $\left(\mathrm{SO}_{4}\right)^{--}$produced when the acid dissociates in water, compress the water molecules by their electrostatic attraction and reduce the compressibility of the solution. On the other hand, the curves of total vapor pressure versus concentration shown in Fig. $7^{*}$, indicate negative deviations from their ideal behaviors.

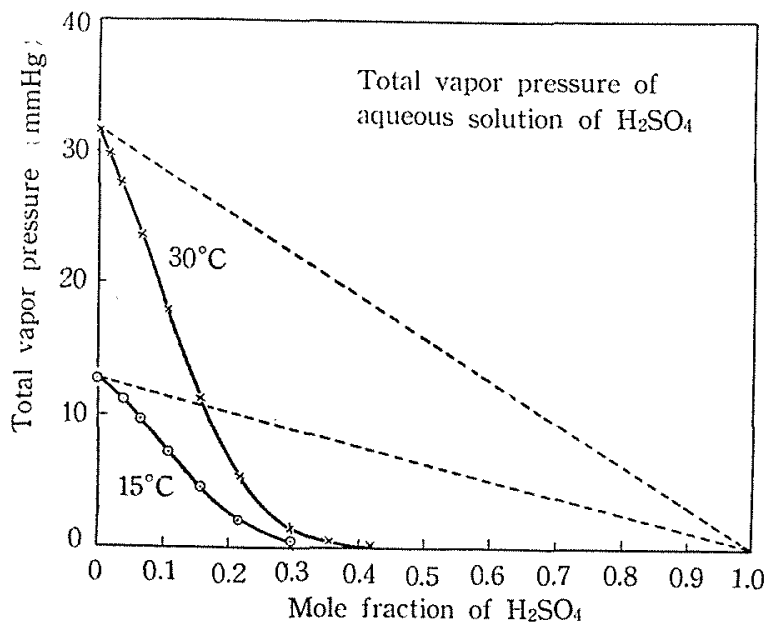

Fig. 7. Total vapor pressure versus concentration of aqueous solution of $\mathrm{H}_{2} \mathrm{SO}_{4}$.

* This figure was drawn from the data given in International Critical Tables, Vol. 3 (McGrawHill, 1928). 
In a special case when one component is water in non-ideal solution, the problem becomes more complicated on account of the abnormality of water. If we seek the cause for negative deviation of compressibility of aqueous solution of sulfuric acid only in the intermolecular forces, including ionic attraction, it might be natural that the curves corresponding to two temperatures shift parallel to each other. And, since the compressibility is reduced at the lower temperature on account of the increase in intermolecular force, the entire curve at $15^{\circ} \mathrm{C}$ would lie below the curve at $30^{\circ} \mathrm{C}$. But, in fact, both curves intersect in the neighborhood of the concentration of $0.1 \mathrm{mf}$ and the curve at $15^{\circ} \mathrm{C}$ rises above the curve at $30^{\circ} \mathrm{C}$ within the concentration of less than $0.1 \mathrm{mf}$, as already seen in Fig. 4 .

The fact can be explained by the association of water molecules. That the compressibility of pure water at $15^{\circ} \mathrm{C}$ is greater than that at $30^{\circ} \mathrm{C}$ is accounted for by the fact that the degree of association of water molecules is larger at the lower temperature. And the compressibility of the solution falls rapidly on account of the depolymerization of water molecules by the sulfuric acid added.

Hereupon, we must assume both the intermolecular forces and the depolymerization of water molecules to be causes for decrease in the compressibility in the lower concentration region. We try to evaluate the respective degree of contributions of these two to the decrease in the compressibility.

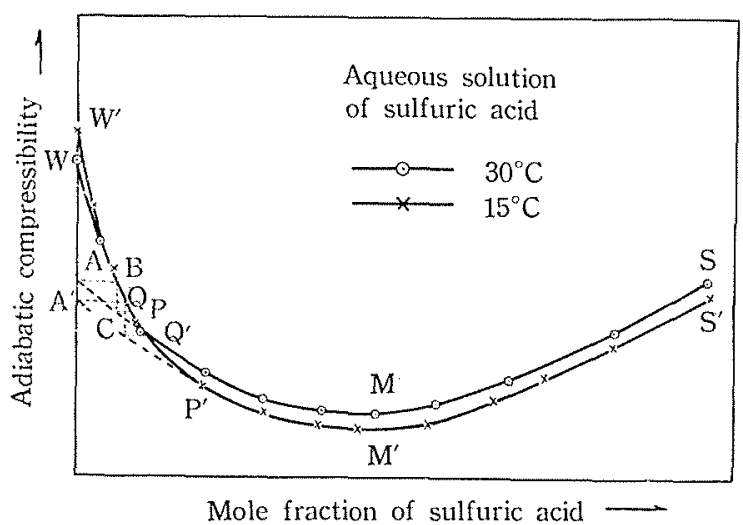

Fig. 8. Extrapolation of compressibility versus concentration curves.

The compressibility-curves have large gradients at the concentration lower than $0.1 \mathrm{mf}$. We extrapolate naturally the parts of the curves higher than $0.1 \mathrm{mf}$ and obtain the curves SMPA, $\mathrm{S}^{\prime} \mathrm{M}^{\prime} \mathrm{P}^{\prime} \mathrm{A}^{\prime}$ shown in Fig. 8. The curves are considered to show the negative deviation originating from the intermolecular force. Therefore, if water would be a non-associated liquid, the points $A$ and $A^{\prime}$ might give the values 
of compressibility of pure water at $30^{\circ} \mathrm{C}$ and $15^{\circ} \mathrm{C}$ respectively. Accordingly, the lowering of compressibility by the depolymerization can be estimated to be

and

$$
\mathrm{WA}=9 \times 10^{-12}\left[\mathrm{~cm}^{2} / \text { dyne }\right] \text { at } 30^{\circ} \mathrm{C} \text {, }
$$$$
W^{\prime} A^{\prime}=13 \times 10^{-12}\left[\mathrm{~cm}^{2} / \text { dyne }\right] \text { at } 15^{\circ} \mathrm{C} \text {. }
$$

Next, we will determine the concentration at which the association of water molecules are completely broken by the sulfuric acid added. In order to get the value at $30^{\circ} \mathrm{C}$, we draw in Fig. 8 a straight line parallel to the abscissa from point $A$ as far as point B at which it intersects the curve WPM, and then draw a straight line parallel to the ordinate from $B$ till point $C$ at which it intersects the curve APM, and finally, draw a straight line parallel to the abscissa from $\mathrm{C}$ till point $\mathrm{Q}$ at which it intersects the curve WPM again. The concentration of $0.085 \mathrm{mf}$ corresponding to the point $Q$ is the desired one, because at the point $B$ which corresponds to the decrease WA, the decrease $\mathrm{BC}$ by the effect of intermolecular force must be added and the point $\mathrm{Q}$ which corresponds to the sum $\mathrm{CW}$ should be a desired point. Similarly, we get the concentration of $0.11 \mathrm{mf}$ which corresponds to the point $\mathrm{Q}^{\prime}$.

There are two evidences as given below which support the deduction that the association of water molecules in aqueous solution of sulfuric acid is completely broken up to the concentration of $0.085 \mathrm{mf}$ at $30^{\circ} \mathrm{C}$ and up to $0.11 \mathrm{mf}$ at $15^{\circ} \mathrm{C}$.

The first evidence: The velocity-temperature characteristic shown in Fig. 5 can be rewritten into the compressibility-temperature characteristic shown in Fig. 9. In this figure, the straight line III at the concentration of $0.0963 \mathrm{mf}$ shows that the compressibility increases with temperature. In almost all liquids except water, it is known that the compressibility increases with temperature. The feature shows that the liquid is non-associated. Therefore, it can be deduced that the association of water molecules has been broken by the sulfuric acid added until the concentration becomes $0.0963 \mathrm{mf}$.

The second evidence: Studies on ice (11) show that the associated water molecules form tetrahedra, each molecule having four neighbors, and such tetrahedra connected form lattice. In water, the $\mathrm{X}$-ray measurements offer the strong evidence in favor of widespread local tetrahedral arrangement at room temperature and below. On the other hand, at the concentration of $0.1 \mathrm{mf}$ which is approximately the mean of $0.085 \mathrm{mf}$ and $0.11 \mathrm{mf}$, the ratio of water molecules to $\mathrm{H}_{2} \mathrm{SO}_{4}$ molecules is $9: 1$. Since a molecule of $\mathrm{H}_{2} \mathrm{SO}_{4}$ emits two protons, the ratio of $\mathrm{H}_{2} \mathrm{O}$ molecules to protons is $9: 2$. As the mechanism by which the association of water molecules is broken, we assume that when a proton from sulfuric acid catches a water molecule to form a hydronium ion $\left(\mathrm{H}_{3} \mathrm{O}\right)^{+}$, the associated group is broken down to sub-groups or to 
isolated molecules. From the above ratio, the number of water molecules assigned to a proton is 4.5. Since the minimum size of regular group of associated molecules is considered to be a tetrahedron consisting of five molecules, there exist almost sufficient protons to destroy all groups of associated molecules at the concentration of $0.1 \mathrm{mf}$.

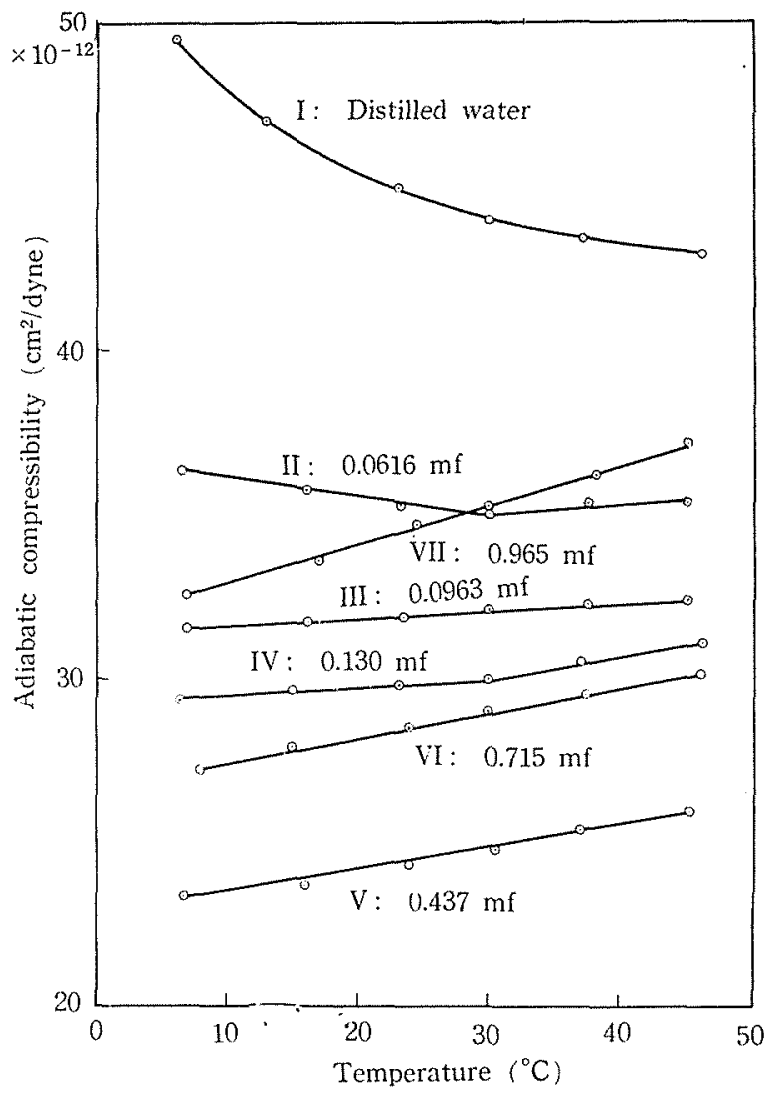

Fig. 9. Compressibility versus temperature, with concentration as parameter.

\section{Regarding the compressibility-temperature characteristic}

The characteristics are shown in Fig. 9. The curve I shows that the compressibility of the distilled water falls with temperature. This is a well-known fact. On the contrary, the compressibility of the aqueous solution of sulfuric acid of higher concentration rises almost linearly with temperature, as known from straight lines $\mathrm{V}$, VI, VII. The aqueous solution is said to be a normal liquid. Water is an abnormal liquid. The abnormality rises from the association of the molecules. An interesting fact is seen in curve II. The curve consists of two straight lines, one of which has a negative gradient below the temperature $30^{\circ} \mathrm{C}$ and the other has a positive gradient 
above $30^{\circ} \mathrm{C}$. The part of negative gradient means that the association of water molecules remains, while the part of positive gradient means that association has vanished, that is, $30^{\circ} \mathrm{C}$ is a critical temperature of the solution of the concentration of $0.0616 \mathrm{mf}$. As already mentioned, all of the associations are considered to be destroyed in the solution of concentration of $0.0963 \mathrm{mf}$, as known from the positive gradient of the straight line III.

\section{Regarding the pattern of the velocity-temperature curves}

Returning to Fig. 5, we want to point out a feature which can be seen in the family of curves. Since the curve III is a straight line parallel to the abscissa, the curve II a combination of a straight line parallel to the abscissa and a straight line of positive gradient, and the curve IV a combination of a straight line parallel to the abscissa and a straight line of negative gradient, the hypothetical velocity. temperature curves shown in Fig. 10 occurs to us. If the measurement would be done in the wider temperature range, the curve III would be a straight line of negative gradient in the higher temperature, and a straight line of positive gradient in the lower temperature, and it would become trapezoidal as a whole. All of the

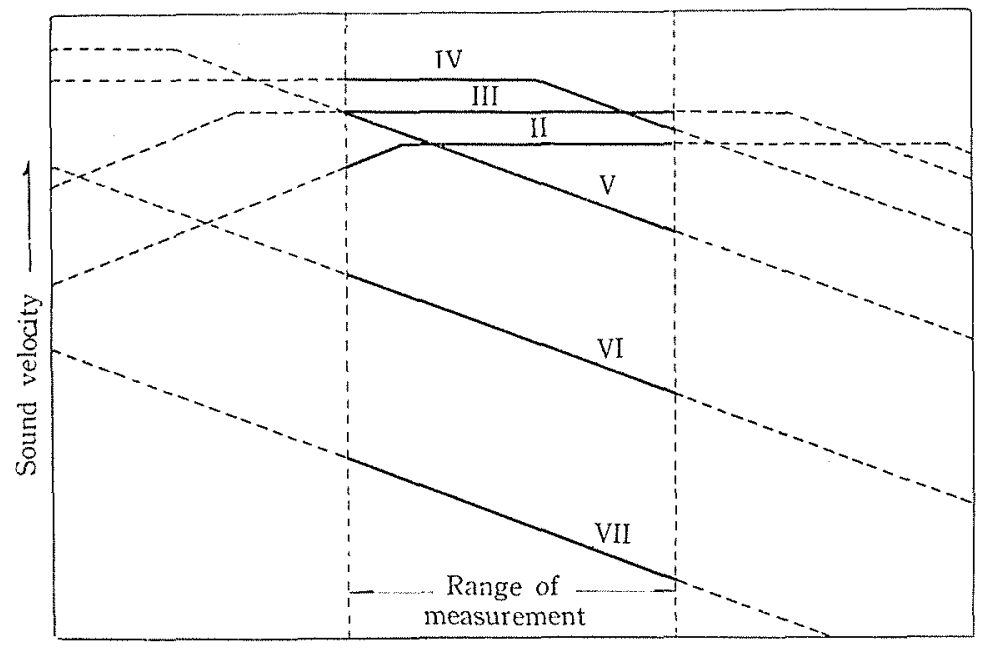

Temperature

Fig. 10. Hypothetical velocity versus temperature curves.

straight lines which appear in Fig. 5 are imagined to be parts of a trapezoid which is the hypothetical entire curve. And it is seen that the trapezoid shifts to the lower temperature as the concentration increases. Willard (12) has stated that such a hypothetical velocity-temperature curve is parabolic in the mixtures of water-ethanol and of water-other organic liquids. But, by the author's measurement, the curve is trapezoidal but not parabolic in the mixture of sulfuric acid and water. 


\section{Acknowledgement}

Having been supported by a scholarship of the Ministry of Education in 1957, the present research was performed in Department of Physics, Faculty of Science, Kyoto University. The author wishes to thank Professor I. Takahashi for his continual encouragement and valuable discussions. His thanks are also due to Professor M. Ishibashi and Mr. Y. Yamamoto of the same university for their kind guidance in the technique of acidimetry.

\section{REFERENCES}

1. P. Prosorow: Zh. Fiz. Kim. 14 (1940), 391. (See B. B. KudrJawzew: Anvendung von Ultraschallverfahren bei Physikalisch-Chemischen Untersuchungen, (Deutscher Verlag der Wissenschaften, Berlin, 1955, translated from the Russian edition (1952)).

2. I. TAkahashi, Y. IshidA and M. OTA: Mem. Coll. Sci. Univ. Kyoto, A 27 (1953), 41.

3. I. TAKAHASHI and Y. Ishida: J. Appl. Phys. Japan, 25 (1956), 247 (in Japanese).

4. Y. Ishidd: Sci. Report, Shimane Univ., 7 (1957), 30.

5. P. Debye and F. W. Sears: Proc. Nat. Acad. Sci., 18 (1932), 409.

6. I. G. Mikhallov: C. R. (Doklady) URSS 31 (1941), 324.

7. I. G. Mikhallov: Ibid. 26 (1940), 147.

8. I. G. Mikfiallov: Ibid. 31 (1941), 550. (Concerning the last three, see O. Nomoto: Chônpa, (Kawade-Shobô, Tokyo, 1948)).

9. A. W. Smith and L.M. Ewing: J. Chem. Phys., 7 (1939), 632.

10. P. J. Ernst : J. Acous. Soc. America, 19 (1947), 372.

11. R. W. GurNeY: Ionic Processes in Solutions, (McGraw-Hill) p. 48.

12. G. W. Willard: J. Acous. Soc. America, 19 (1947), 235. 